\title{
BMJ Open Effect of intratracheal dexmedetomidine administration on recovery from general anaesthesia after gynaecological laparoscopic surgery: a randomised double-blinded study
}

Fei Wang, ${ }^{1}$ Haoxiang Zhong, ${ }^{1}$ Xiaoyan Xie, ${ }^{1}$ Weiping Sha,${ }^{1}$ Caili Li, ${ }^{1}$ Zhenping Li, ${ }^{1}$ Zhuomei Huang, ${ }^{1}$ Chaojin Chen ${ }^{2}$

To cite: Wang $\mathrm{F}$, Zhong $\mathrm{H}$, Xie X, et al. Effect of intratracheal dexmedetomidine administration on recovery from general anaesthesia after gynaecological laparoscopic surgery: a randomised doubleblinded study. BMJ Open 2018;8:e020614. doi:10.1136/ bmjopen-2017-020614

- Prepublication history and additional material for this paper are available online. To view these files, please visit the journal online (http://dx.doi. org/10.1136/bmjopen-2017020614).

FW and ZH contributed equally.

Received 13 November 2017 Revised 15 February 2018 Accepted 23 February 2018

Check for updates

${ }^{1}$ Department of Anesthesiology and Pain Medicine, Guangdong Medical College, Huizhou First People's Hospital, Huizhou, Guangdong, China

${ }^{2}$ Department of Anesthesiology, The Third Affiliated Hospital of Sun Yat-sen University, Guangzhou, Guangdong, China

Correspondence to Dr Chaojin Chen; chenchaojin_sysu@foxmail.com and $\mathrm{Dr}$ Zhuomei Huang;

huangzhuomei316@163.com

\section{ABSTRACT}

Objectives To examine the efficacy of intratracheal dexmedetomidine (Dex) injection for the prevention of the laryngeal response on emergence from general anaesthesia following gynaecological laparoscopic surgery. Design Prospective, randomised, double-blinded, controlled trial.

Setting A general hospital, Guangdong Province, China. Participants All patients who underwent elective laparoscopic gynaecological surgery, aged 18-60 years old, 40-80 kg in weight, American Society of Anesthesiologists class I-II were eligible. Patients were excluded if they had respiratory disease, heart disorders which might represent risk factors of potential complications of Dex such as bradycardia, heart block, coronary heart disease, uncontrolled hypertension or the long-term use of sedative drugs.

Intervention Patients were randomly allocated to either receive intratracheal Dex (DT), intravenous Dex (DV) or intravenous saline (CON, $n=30$, respectively). In the DT and DV groups, Dex $(0.5 \mu \mathrm{g} / \mathrm{kg})$ was diluted and mixed in 1 or $20 \mathrm{~mL}$ of saline, respectively, and injected via the intratracheal or intravenous route $30 \mathrm{~min}$ before the completion of the surgery.

Outcome measures The primary outcome was the coughing extent among the three groups. Secondary outcomes included awareness time, extubation time, postoperative visual analogue scale and Steward recovery score.

Results Compared with the CON group, the extent of coughing was significantly reduced in both the DV group and the DT group. Furthermore, the mean time to awareness (13.4 (4.3) vs $8.8(2.9), p<0.001)$ and the extubation time (14.3 (4.3) vs 8.4 (3.6), $p<0.001$ ) were reduced in the DT group. Patients in the DT group also experienced better early recovery quality and less pain than those in the CON group. Furthermore, intratracheal Dex administration contributed to improved stability in haemodynamics with no significant side effects.

Conclusions Intratracheal Dex administration may avoid untoward laryngeal responses for patients emerging from general anaesthesia after gynaecological laparoscopy. Trial registration number ChiCTR-IOR-15007611.

\section{Strengths and limitations of this study}

- This is the first trial to explore the efficacy of intratracheal dexmedetomidine (Dex, $0.5 \mu \mathrm{g} / \mathrm{kg}$ ) administration to attenuate or avoid untoward laryngeal reflexes in patients emerging from general anaesthesia after gynaecological laparoscopy.

- Intratracheal Dex injection may attenuate laryngeal reflexes, provide better recovery quality and less pain and improve haemodynamic stability with no significant side effects.

- All participants were recruited from a single hospital, which may limit the generalisability of the study's results.

- The absorption characteristics of intratracheal Dex administration still require further exploration.

\section{INTRODUCTION}

The incidence of intratracheal tube-induced laryngeal irritation, including coughing, bucking and cardiovascular stimulation, on emergence from general anaesthesia has been reported to occur in $38 \%$ and $96 \%$ of cases. ${ }^{1}$ This may cause agitation and unstable haemodynamics during anaesthesia recovery, and can lead to complications, such as laryngeal oedema, sore throat, increased intra-abdominal pressure and anastomotic bleeding. ${ }^{2}$ Accordingly, many anaesthesiologists have been seeking methods through which such laryngeal irritation can be attenuated, thus allowing for a smoother extubation. ${ }^{3}$ This is particularly appealing for laparoscopic surgery, as pneumoperitoneum and hypercapnia can result in sympathetic excitement and an increase in blood pressure and heart rate (HR) during surgery. ${ }^{4}$ Furthermore, it has been reported that hypercapnia might contribute to the aggravation of cough reflexes. $^{5}$ 
Several strategies have been reported to facilitate a smoother extubation, such as intravenous lidocaine, ${ }^{1}$ remifentanil and dexmedetomidine (Dex). ${ }^{6}$ Dex is a potent, alpha-2-selective adrenoceptor agonist that causes sympatholysis, sedation and analgesia without respiratory compromise. ${ }^{7}$ Delivering Dex intravenously to attenuate airway and circulatory reflexes during extubation in adults has been extensively evaluated in clinical studies, including studies in the laparoscopic surgery setting. ${ }^{8}$ Recent studies have also indicated that the intranasal application of Dex is significantly useful and effective in the achievement of improved sedation and analgesia. ${ }^{9}$ Additionally, intratracheal local anaesthetic instillation has been reported to be effective in preventing laryngeal reflexes. ${ }^{10}$ We previously reported that intratracheal administration of medication has been widely used in clinical practices. ${ }^{11}$ However, both the efficacy and safety profile of intratracheal Dex application are largely unknown and the technique has not been specifically investigated.

We conducted a prospective, randomised, doubleblinded, controlled study to examine the efficacy of intratracheal injection of Dex before tracheal extubation for the prevention of the bucking response on emergence from general anaesthesia, compared with intravenous Dex and placebo administration.

\section{METHODS}

\section{Study subjects}

In accordance with the Declaration of Helsinki, this randomised controlled trial was approved by the Institutional Review Board of the First People's Hospital of Huizhou City (approval number: 2015006) and was registered at www.chictr.org on 11 October 2015 (registration number: ChiCTR-IOR-15007611).

All patients undergoing elective laparoscopic gynaecological surgery in the hospital between February and May 2016 were considered for inclusion. Patients who were between 18 and 60 years of age, weighed between 40 and $80 \mathrm{~kg}$ and had been designated as American Society of Anesthesiologists (ASA) physical status (PS) I and II using the ASA PS scoring system were prospectively enrolled in this study. Patients were excluded from the study in the event of respiratory disease, heart disorders which might represent risk factors of potential complications of Dex such as bradycardia, heart block, sick sinus syndrome and coronary heart disease, hepatic or renal insufficiency, uncontrolled hypertension or long-term use of sedative drugs, such as benzodiazepines, barbiturates and other sedative hypnotics. Patients who were transferred to open surgery during the operation were excluded from the final analysis.

\section{Group allocation}

Written informed consent was obtained from all patients. Patients were randomly allocated to the Dex intratracheal injection group (DT group), the Dex intravenous injection group (DV group) or the control group (CON group). Randomisation was performed by an independent investigator based on a random number generation table in Microsoft Excel. Details of group allocation were all stored in numbered, opaque and sealed envelopes. All the patients, investigators, anaesthesiologists and statisticians were blinded to group assignment. Group allocation was not revealed until the final statistical analysis was completed.

\section{Sample size}

Based on retrospective results from the same surgical group, the cough score (mean (SD)) in the CON group was $2.2(0.8)$ and was reduced to $1.7(0.8)$ in response to the intravenous administration of Dex. With statistical significance set to $5 \%$ (two sided) and assuming a power of $90 \%$ and setting the cough score as the primary variable, we determined that a sample size of 27 was necessary for each group. In order to allow for a potential dropout rate of $10 \%, 30$ patients were finally enrolled in each group.

\section{Procedure}

To eliminate any possible effects of variation in the surgical technique used, all procedures were undertaken by the same surgical group. On arrival at the operating room, an IntelliVue MP30-type monitor (Philips, Netherlands) was used to record the systolic blood pressure (SBP), the diastolic blood pressure (DBP), the mean blood pressure (MBP) and the HR; the bispectral index (BIS) was also measured using an Aspect A-2000 monitor (Draeger, Germany).

Anaesthesia was induced through intravenous injection of $0.1 \mathrm{mg} / \mathrm{kg}$ midazolam, $2 \mathrm{mg} / \mathrm{kg}$ propofol and $0.4 \mu \mathrm{g} / \mathrm{kg}$ sufentanil, followed by $0.2 \mathrm{mg} / \mathrm{kg}$ cisatracurium. Three minutes later, intratracheal intubation was carried out using an enhancement type $\square$ intratracheal tube (Xi'an Shen Lan Biomedical Engineering, approval number: 37140501) with an internal diameter of $7.0 \mathrm{~mm}$. For maintenance of anaesthesia, sevoflurane was used in $100 \%$ oxygen $\left(\mathrm{O}_{2}\right)$ with an infusion of $2 \mu \mathrm{g} / \mathrm{kg} / \mathrm{min}$ cisatracurium and $0.04 \mu \mathrm{g} / \mathrm{kg} / \mathrm{min}$ remifentanil to maintain a target BIS of 40-60. Mechanical ventilation was adjusted to maintain an end-tidal carbon dioxide pressure $\left(\mathrm{P}_{\mathrm{ET}} \mathrm{CO}_{2}\right)$ of $34-45 \mathrm{~mm} \mathrm{Hg}$.

Thirty minutes before the end of the surgery, cisatracurium was discontinued and $0.1 \mathrm{mg} / \mathrm{kg}$ tropisetron was administered in order as prophylaxis for postoperative nausea and vomiting (PONV). According to an earlier report, ${ }^{12}$ Dex $(0.5 \mu \mathrm{g} / \mathrm{kg}$, diluted and mixed in $1 \mathrm{~mL}$ saline in a medical spray bottle) was sprayed down the intratracheal tube of patients in the DT group, and the patients in the DV group were intravenously given the same dose of Dex (diluted and mixed in $20 \mathrm{~mL}$ saline) as an infusion over $10 \mathrm{~min}$. Total body weight of patients was taken into consideration to calculate the dose of Dex. Patients in the CON group were intravenously injected with saline $(20 \mathrm{~mL})$ instead of Dex for the same period 


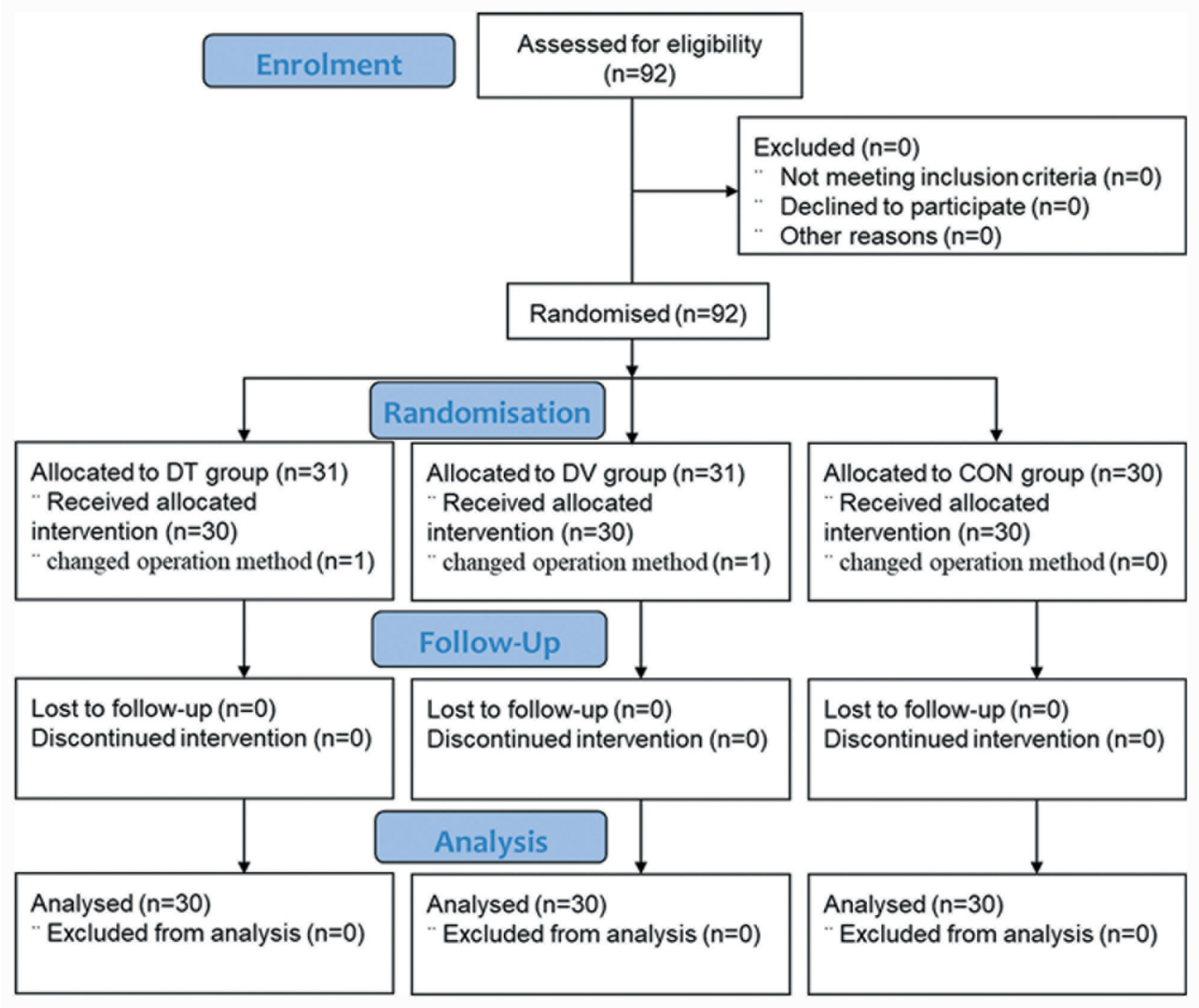

Figure 1 Consolidated Standards of Reporting Trials (CONSORT) flow diagram. CON, control group; DT, Dex intratracheal injection group; DV, Dex intravenous injection group.

of time. Patients in the three groups received continuous sevoflurane inhalation and remifentanil infusion until all surgical procedures were completed, and the $\mathrm{O}_{2}$ flow was increased to $5 \mathrm{~L} / \mathrm{min}$ to exclude sevoflurane. Another anaesthesiologist, blinded to the randomisation, performed the remaining anaesthetic recovery processes and collected all the data.

\section{Data collection}

We recorded a range of demographic and intraoperative variables including age, weight, the pneumoperitoneum time and pressure, the duration of anaesthesia and operation, the time to awareness (from the end of the surgery to follow verbal command) and the time to extubation (from the end of the surgery to extubation). SBP, DBP, MBP and HR values were recorded 5 min before anaesthesia (T0), immediately after the administration of Dex (T1), 5 min after the administration of Dex (T2), 10 min after the administration of Dex (T3), at the end of surgery (T4), at the point of awareness (T5), at the point of extubation (T6), $2 \mathrm{~min}$ after extubation (T7), $5 \mathrm{~min}$ after extubation (T8), $15 \mathrm{~min}$ after extubation (T9) and 30 min after extubation (T10). Airway pressure and $\mathrm{SpO}_{2}$ values were recorded at T1, T2, T3 and T4. Subsequently, postoperative pain scores (using the visual analogue scale $(\mathrm{VAS}))$ and the Steward recovery score $(\mathrm{SRS})^{13}$ were assessed at T8, T9 and T10.
The primary outcome was the coughing severity, as judged by the 4-point scale as reported previously, ${ }^{1}$ which was evaluated during the recovery period from the time of awareness to $5 \mathrm{~min}$ after extubation: $0=$ no cough, $1=$ minimal (single) cough, $2=$ moderate $(\leq 5 \mathrm{~s})$ cough and $3=$ severe $(>5 \mathrm{~s})$ cough (bucking). Grades 2 and 3 were considered to be clinically deleterious. The secondary outcomes included the awareness time, the extubation time, the postoperative VAS score, the SRS and the PONV. Bradycardia, tachycardia, hypertension and hypotension were recorded and compared. Bradycardia and tachycardia were defined as an HR $<50$ or $>100$ beats/ min. Hypertension and hypotension were defined as an $\mathrm{MBP}<30 \%$ or $>30 \%$ from the baseline (T0) for $5 \mathrm{~min}$.

\section{Statistical analysis}

Data analysis was performed using SPSS for Windows V.16.0 (SPSS). A one-sample Kolmogorov-Smirnov test was used to test the normality of continuous data. The quantitative variables, which were normally distributed, were expressed as a mean (SD) and analysed by one-way analysis of variance (ANOVA). Haemodynamic parameters among the three groups were tested using ANOVA followed by post hoc analysis. Non-normal data, such as the SRS and pneumoperitoneum pressure, were analysed by the Wilcoxon rank-sum test. Qualitative data were presented as numbers/percentages, and analysed by the 
Table 1 Perioperative and intraoperative baseline variables

\begin{tabular}{|c|c|c|c|c|}
\hline Variables & CON group & DV group & DT group & $P$ values \\
\hline \multicolumn{5}{|l|}{ Preoperative characteristics } \\
\hline Age (years) & $38.3(9.2)$ & $40.2(10.6)$ & $40.7(10.1)$ & 0.621 \\
\hline ASA grade, $n(\%)$ & & & & 0.875 \\
\hline 1 & $5(16.7)$ & 7 (23.3) & $5(16.7)$ & \\
\hline \multicolumn{5}{|l|}{ Intraoperative variables } \\
\hline Anaesthesia time (min) & $97.8(18.5)$ & $92.3(13.2)$ & $95.8(13.8)$ & 0.378 \\
\hline Operation time (min) & $87.8(17.6)$ & $84.5(12.5)$ & $86.0(13.5)$ & 0.690 \\
\hline Pneumoperitoneum time (min) & $74.9(17.7)$ & $73.8(14.5)$ & $72.3(12.2)$ & 0.789 \\
\hline Pneumoperitoneum pressure $(\mathrm{Pa})$ & $13(13-13)$ & $13(12-13)$ & $13(12-13)$ & 0.247 \\
\hline \multirow{2}{*}{ Tachycardia, n (\%) } & $11(33.3)$ & $2(6.7)$ & & 0.005 \\
\hline & $11(33.3)$ & & $3(10.0)$ & 0.015 \\
\hline \multirow[t]{3}{*}{ Hypertension, n (\%) } & $9(30)$ & $2(6.7)$ & $2(6.7)$ & 0.016 \\
\hline & $9(30)$ & $2(6.7)$ & & 0.020 \\
\hline & $9(30)$ & & $2(6.7)$ & 0.020 \\
\hline Hypotension, n (\%) & $0(0)$ & $0(0)$ & $0(0)$ & - \\
\hline
\end{tabular}

Values are expressed as a mean (SD) or an absolute number (percentage) or a mean (IQR).

ASA, American Society of Anesthesiologists; CON group, patients were intravenously injected with saline (20 mL) for $10 \mathrm{~min}$; DT group, Dex $(0.5 \mu \mathrm{g} / \mathrm{kg}$, diluted in $1 \mathrm{~mL}$ saline) was sprayed down the patient's intratracheal tube; DV group, patients were intravenously given Dex (0.5 $\mu \mathrm{g} /$ $\mathrm{kg}$, diluted in $20 \mathrm{~mL}$ saline) for $10 \mathrm{~min}$.

Pearson $\chi^{2}$ test or Fisher's exact test. Differences were considered significant when the corrected $\mathrm{p}$ value was less than 0.05 .

\section{RESULTS}

A total of 92 patients were assessed, however in two cases the operative method was changed. Thus, 90 patients were enrolled in the final study between groups DV, DT and $\mathrm{CON}$ ( $\mathrm{n}=30$ per group, figure 1 ). The patients in the three groups were comparable demographically, in terms of age, weight, ASA grade, duration of anaesthesia, operation and pneumoperitoneum, or pneumoperitoneum pressure (table 1).

\section{Primary and secondary outcomes}

The extent of coughing in both the DV group and DT group (both $\mathrm{p}<0.05$, table 2 ) was significantly lower than the cough score in the CON group. Furthermore, the number of grade 2 and 3 patients accounted for $30 \%$ $(9 / 30)$ of patients in the CON group, which was a much higher proportion than those in the DV group $(10 \%$, $3 / 30)$ and the DT group $(6.7 \%, 2 / 30)$.

Consistent with the intravenous application of Dex, the intratracheal administration of Dex also significantly reduced the mean awareness time and the extubation time when comparing the DT group and the CON group (both $\mathrm{p}<0.001$; table 2). The incidence of PONV did not differ between the three groups.

In terms of SRS, we found that patients in the DT group and the DV group had a better quality of early recovery than those in the CON group at T8 and T9 $(\mathrm{p}<0.001$, table 3). However, at T10, there was no significant difference in the SRS values between the three groups.

The VAS data recorded for the three groups are shown in table 3 and online supplementary table 1 . Significant reductions were observed in both the DT group (2.8 (0.8) vs $3.4(0.6), \mathrm{p}=0.005)$ and DV group (2.6 (0.8) vs $3.4(0.6), \mathrm{p}<0.001)$, compared with the CON group at T8 (table 3$)$. In addition, less patients in the DV (13.3\%) and DT $(16.7 \%)$ groups experienced moderate pain (VAS 4-6) than those in the CON group (46.7\%) at T8 (online supplementary table 1 ).

In terms of respiratory parameters, we found that the intratracheal application of Dex (DT group) had no significant influence on the recorded airway pressure or the $\mathrm{SpO}_{2}$, when compared with either the CON group or the DV group (all $\mathrm{p}>0.05$, figure 2 ).

\section{Haemodynamic changes}

Compared with the CON group, perioperative mean arterial pressure values in the DT group and the DV group showed less dramatic variation (figure 3A), particularly at 


\begin{tabular}{|c|c|c|c|c|}
\hline Variables & $\begin{array}{l}\text { CON } \\
\text { group }\end{array}$ & DV group & DT group & $\begin{array}{l}P \\
\text { values }\end{array}$ \\
\hline \multirow{3}{*}{$\begin{array}{l}\text { Coughing } \\
\text { extent } \\
(n, 0 / 1 / 2 / 3)\end{array}$} & $6 / 15 / 6 / 3$ & $18 / 9 / 3 / 0$ & $16 / 12 / 2 / 0$ & 0.008 \\
\hline & 6/15/6/3 & $18 / 9 / 3 / 0$ & & 0.005 \\
\hline & $6 / 15 / 6 / 3$ & & $16 / 12 / 2 / 0$ & 0.01 \\
\hline \multirow{3}{*}{$\begin{array}{l}\text { Awareness } \\
\text { time (min) }\end{array}$} & $13.4(4.3)$ & $8.4(5.0)$ & $8.8(2.9)$ & 0.000 \\
\hline & $13.4(4.3)$ & $8.4(5.0)$ & & 0.000 \\
\hline & $13.4(4.3)$ & & $8.8(2.9)$ & 0.000 \\
\hline \multirow{3}{*}{$\begin{array}{l}\text { Extubation } \\
\text { time (min) }\end{array}$} & $14.3(4.3)$ & $7.7(3.1)$ & $8.4(3.6)$ & 0.000 \\
\hline & $14.3(4.3)$ & $7.7(3.1)$ & & 0.000 \\
\hline & $14.3(4.3)$ & & $8.4(3.6)$ & 0.000 \\
\hline PONV & $1(3.3)$ & $0(0)$ & $1(3.3)$ & 0.439 \\
\hline
\end{tabular}

Values are expressed as a mean (SD) or an absolute number (percentage). Coughing severity was evaluated during the recovery period from the time of awareness to 5 min after extubation using the 4-point scale: $0=$ no cough, $1=$ minimal (single) cough, $2=$ moderate $(\leq 5 \mathrm{~s})$ cough, $3=$ severe $(>5 \mathrm{~s})$ cough. PONV was noted if the patient felt nauseous or vomited after surgery. CON group, patients were intravenously injected with saline $(20$ $\mathrm{mL})$ for $10 \mathrm{~min}$; DT group, Dex $(0.5 \mu \mathrm{g} / \mathrm{kg}$, diluted in $1 \mathrm{~mL}$ saline) was sprayed down the patient's intratracheal tube; DV group, patients were intravenously given Dex $(0.5 \mu \mathrm{g} / \mathrm{kg}$, diluted in $20 \mathrm{~mL}$ saline) for $10 \mathrm{~min}$; PONV, postoperative nausea and vomiting.

the time of awareness (T5), the time of extubation (T6) and $2 \mathrm{~min}$ after extubation (T7). Similarly, the HR values showed less variation at the time of awareness (T5), the time of extubation (T6) and $2 \mathrm{~min}$ after extubation (T7; figure $3 \mathrm{~B}$ ) in the DV group.

The number of cases experiencing tachycardia during tracheal extubation in the DT group (2/30 vs $11 / 30$, $\mathrm{p}=0.005)$ and the DV group ( $3 / 30$ vs $11 / 30, \mathrm{p}=0.015$, table 1) was significantly lower than those recorded in the CON group. There were nine patients with hypertension in the CON group and two patients with hypertension in both the DT and the DV groups during the recovery period, with significant intergroup differences $(9 / 30$ vs $2 / 30$, both $\mathrm{p}=0.020$ ).

There were no statistical differences in the proportion of patients with bradycardia and hypotension between the DT group, the DV group and the CON group (table 1).

\section{DISCUSSION}

The results of this study show that intratracheal administration of Dex reduced the extent of coughing observed in patients after gynaecological laparoscopic surgery at the time of extubation when compared with salinetreated control group. Furthermore, the intratracheal application of Dex did not demonstrate any negative airway events in the present study population, producing stable haemodynamic effects, improving the quality of
Table 3 The SRS and VAS scores among the three groups

\begin{tabular}{lllll}
\hline & $\begin{array}{l}\text { CON } \\
\text { group }\end{array}$ & DV group & DT group & P values \\
\hline SRS (T8) & $4(3-4)$ & $4(4-5)$ & $4(4-5)$ & 0.001 \\
& $4(3-4)$ & $4(4-5)$ & & 0.005 \\
& $4(3-4)$ & & $4(4-5)$ & 0.000 \\
\hline SRS (T9) & $5(4-5)$ & $5(4-5)$ & $5(5-5)$ & 0.001 \\
& $5(4-5)$ & $5(4-5)$ & & 0.005 \\
& $5(4-5)$ & & $5(5-5)$ & 0.000 \\
\hline SRS (T10) & $6(5-6)$ & $6(6-6)$ & $6(6-6)$ & 0.323 \\
& $6(5-6)$ & $6(6-6)$ & & 0.139 \\
& $6(5-6)$ & & $6(6-6)$ & 0.394 \\
\hline VAS (T8) & $3.4(0.6)$ & $2.6(0.8)$ & $2.8(0.8)$ & 0.000 \\
& $3.4(0.6)$ & $2.6(0.8)$ & & 0.000 \\
& $3.4(0.6)$ & & $2.8(0.8)$ & 0.005 \\
\hline VAS (T9) & $3.1(0.6)$ & $2.6(0.7)$ & $2.7(0.7)$ & 0.015 \\
& $3.1(0.6)$ & $2.6(0.7)$ & & 0.005 \\
\hline & $3.1(0.6)$ & & $2.7(0.7)$ & 0.038 \\
\hline VAS (T10) & $3.2(0.6)$ & $2.9(0.7)$ & $2.6(0.8)$ & 0.009 \\
& $3.2(0.6)$ & $2.9(0.7)$ & & 0.1 \\
\hline & $3.2(0.6)$ & & $2.6(0.8)$ & 0.002 \\
\hline
\end{tabular}

Values are expressed as mean (SD) or median (IQR). The SRS was determined according to consciousness ( $3=$ awake, $2=$ responds to verbal stimuli, $1=$ responds to tactile stimuli, $0=$ not responding), airway condition $(2=$ cough on command or cry, $1=$ maintains good airway, $0=$ requires airway assistance) and motor condition ( $2=$ moves limbs purposefully, $1=$ non-purposeful movement, $0=$ not moving). The VAS score was defined on a scale of 0 (no pain) to 10 (the worst pain imaginable).

CON group, patients were intravenously injected with saline (20 $\mathrm{mL})$ for $10 \mathrm{~min}$; DT group, Dex $(0.5 \mu \mathrm{g} / \mathrm{kg}$, diluted in $1 \mathrm{~mL}$ saline) was sprayed down the patient's intratracheal tube; DV group, patients were intravenously given Dex $(0.5 \mu \mathrm{g} / \mathrm{kg}$, diluted in 20 $\mathrm{mL}$ saline) for $10 \mathrm{~min}$; SRS, Steward recovery score; VAS, visual analogue scale.

early recovery and improving the pain score of patients. These effects are similar to those observed following the intravenous administration of Dex.

Pneumoperitoneum and surgical position during laparoscopic surgery may contribute to the $\mathrm{CO}_{2}$ absorption, the increased intracuff pressure and the aggravation of laryngeal reflexes during anaesthesia recovery. ${ }^{14} 15$ Laryngeal responses, such as bucking and coughing on emergence from anaesthesia, can lead to a variety of complications, such as laryngeal spasm, laryngeal oedema, sore throat, increased intra-abdominal pressure and anastomotic bleeding. ${ }^{1}$ Various interventions have been studied attempting to attenuate this sympathetic response, including the administration of various drugs, such as lidocaine ${ }^{16}$ and short-acting opioids, ${ }^{17}$ before tracheal extubation. The present study found that the intratracheal administration of Dex also represents an effective way to prevent the laryngeal responses observed on emergence from general anaesthesia. 
A

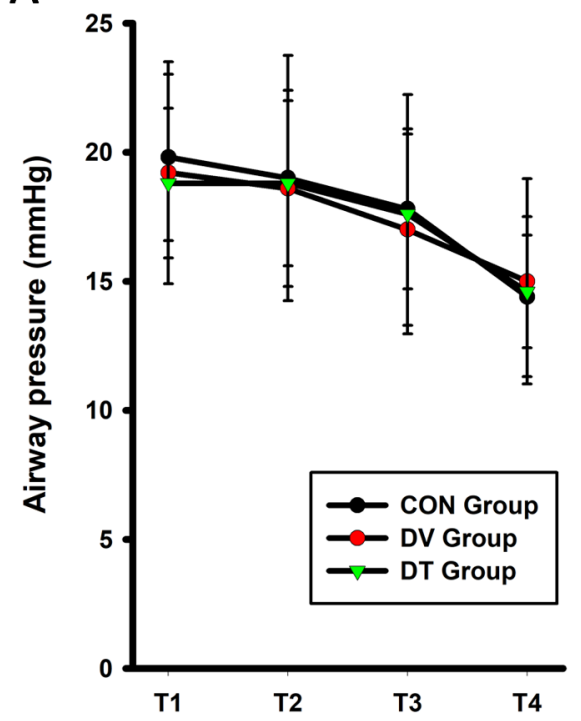

B

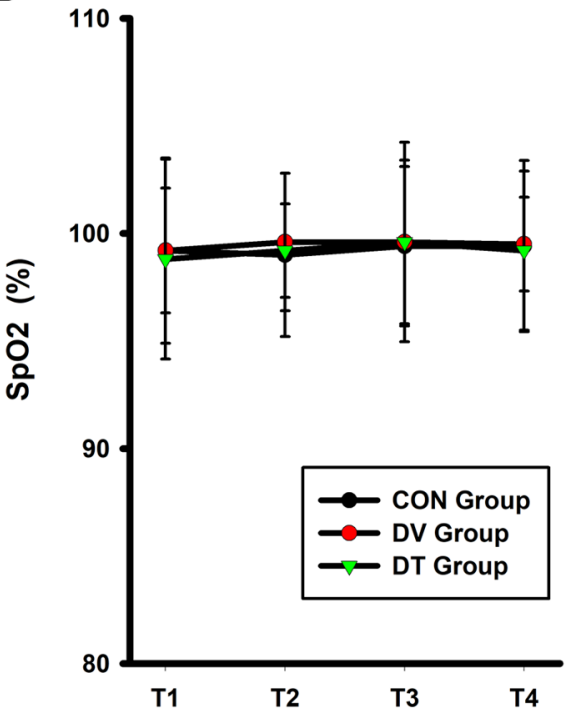

Figure 2 Airway pressure and $\mathrm{SpO}_{2}$ among the three groups. (A) Airway pressure and (B) $\mathrm{SpO}$, among the CON, DT and DV groups. Dex $(0.5 \mu \mathrm{g} / \mathrm{kg})$ was diluted in 1 or $20 \mathrm{~mL}$ of saline, respectively, and injected via the intratracheal (DT group) or intravenous (DV group) route $30 \mathrm{~min}$ before the completion of surgery. In the CON group, patients were intravenously injected with saline $(20 \mathrm{~mL})$ for $10 \mathrm{~min}$. Values are expressed as a mean (SD). $\mathrm{SpO}_{2}$, pulse oxygen saturation; $\mathrm{T} 1$, immediately after the administration of Dex; T2, 5 min after the administration of Dex; T3, 10 min after the administration of Dex; T4, at the end of surgery.

Intratracheal medication has been reported to provide a rapid means of accessing the systemic circulation when intravenous administration is not possible in emergency situations. ${ }^{18}$ Being a non-invasive and highly convenient method, intratracheal administration has been successfully used to deliver many drugs in various clinical settings, such as the use of lidocaine sprays in tracheal intubation. ${ }^{19}$ The intratracheal administration of Dex could represent an alternative method in particular circumstances when an intravenous line is not possible. In addition, it might be more simple and convenient to apply intratracheal Dex than intranasal Dex during general anaesthesia with an oral or nasal intubation. However, few studies have investigated the efficacy and safety of intratracheal Dex administration during recovery from general anaesthesia. Patton $e t a l^{20}$ reported that the lung was a rapid absorption area which is naturally permeable to many small-molecule and therapeutic drugs, and that both hydrophilic and hydrophobic peptides and proteins could be absorbed across the pulmonary epithelium at a molecular weight-dependent speed. Being a hydrophilic small molecule, we believed that Dex was intratracheally absorbed in the present study order to exert its pharmacological effects. Earlier reports have revealed that Dex, at a dose of $0.5 \mu \mathrm{g} / \mathrm{kg}$ for intravenous administration, could significantly improve the recovery profile by reducing the incidence of emergence agitation in children ${ }^{12}$ and adult population. ${ }^{6} 2122$ The current study indicates that a single dose of intratracheal Dex $(0.5 \mu \mathrm{g} / \mathrm{kg})$ exerted the same effect as intravenous application of the same drug, as reflected by a reduction in the extent of coughing and the postoperative pain score without significant side effects or complications. This was the first randomised controlled study to investigate the effects of intratracheal Dex premedication on the recovery of adult patients from general anaesthesia.

These results might be attributable, in part, to the reduction of sympathetic and laryngeal nerve sensitivity during extubation after both intratracheal and intravenous Dex administration. ${ }^{23}$ Intratracheal Dex is believed to be absorbed rapidly through the bronchial and alveolar capillary network to exert its effect, such as inhibiting the increase in HR and blood pressure in response to sympathetic signals. ${ }^{24}$ Furthermore, it has been demonstrated that Dex can reduce the hyperalgesia caused by remifentanil and pain stimulation, and can yield global haemodynamic stability and prevent violent injury to the throat and vocal cord. ${ }^{25-27}$ Notably, previous reports have confirmed the efficacy of intranasal Dex as a sedative premedication to induce a favourable perioperative anxiolysis ${ }^{28}$ and reduce emergence agitation during recovery from general anesthesia. ${ }^{29}$ In addition, Rani $e t$ $a b^{30}$ found that a single dose of Dex, administered intravenously $15 \mathrm{~min}$ prior to extubation, could suppress the airway reflex during recovery from general anaesthesia. Furthermore, being a highly selective alpha-2 adrenoceptor agonist, Dex was reported to activate alpha-2a adrenoceptors which are located in the locus coeruleus and exert hypnotic action. ${ }^{31}$ Thus, along with its sedative effect and potential analgesic effect in the alleviation of postoperative pain, Dex may expedite a smooth and early recovery. ${ }^{32}$

The potential adverse side effects of Dex include bradycardia, hypotension and delayed recovery. ${ }^{33} 34$ None of 


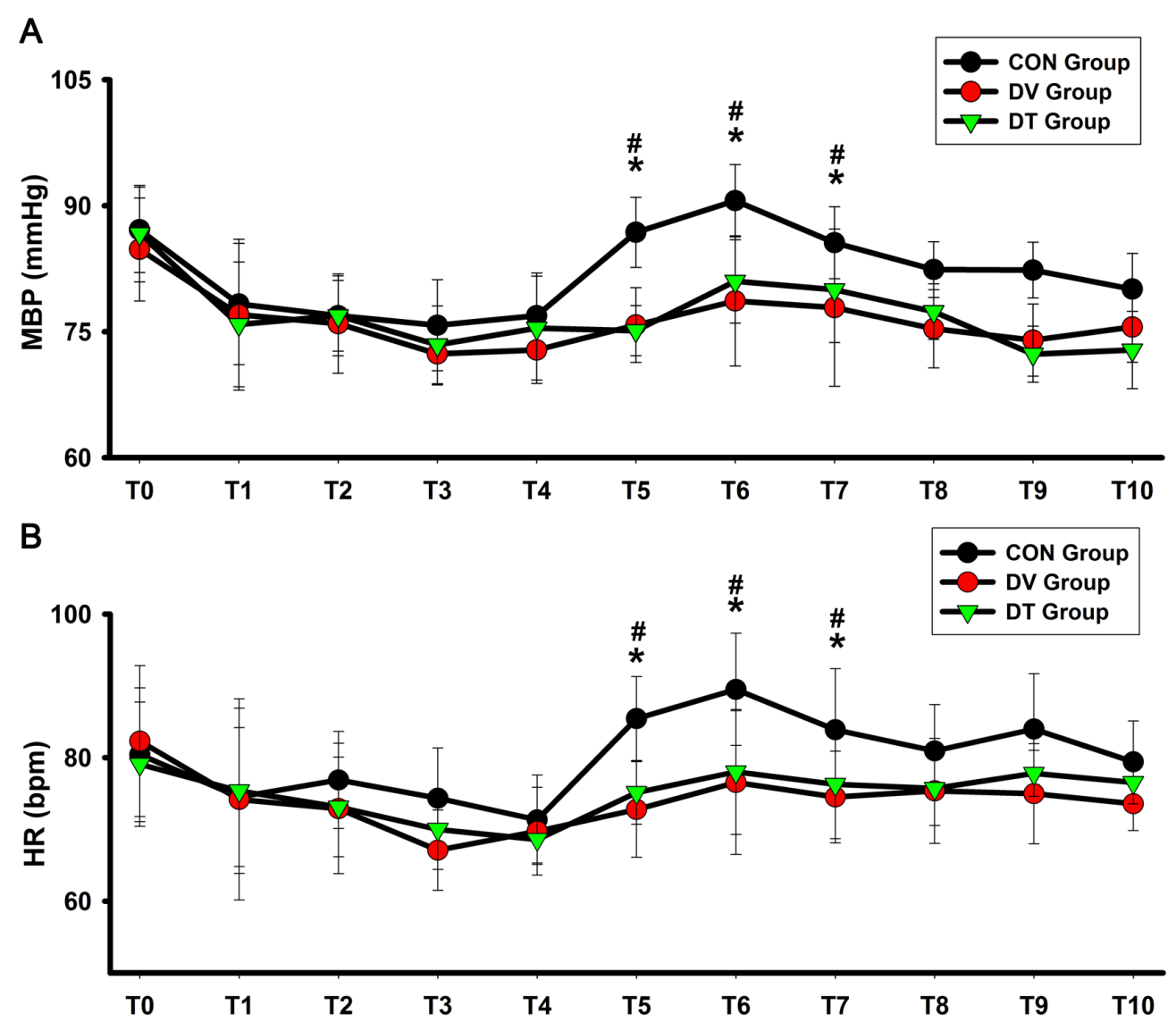

Figure 3 Haemodynamics at different time points. (A) MBP and (B) HR at different time points among the CON, DT and DV groups. Haemodynamics at different time points among the CON, DT and DV groups. Dex $(0.5 \mu \mathrm{g} / \mathrm{kg}) \mathrm{was}$ diluted in $1 \mathrm{or} 20 \mathrm{~mL}$ of saline, respectively, and injected via the intratracheal (DT group) or intravenous (DV group) route 30 min before the completion of the surgery. In the CON group, patients were intravenously injected with saline $(20 \mathrm{~mL})$ for 10 min. Values are expressed as a mean (SD). HR, heart rate; MBP, mean blood pressure; T0, 5 min before anaesthesia; T1, immediately after the administration of Dex; T2, 5 min after the administration of Dex; T3, 10 min after the administration of Dex; T4, at the end of surgery; T5, at the point of awareness; T6, at the point of extubation; T7, 2 min after extubation; T8, 5 min after extubation; T9, 15 min after extubation; T10, 30 min after extubation. ${ }^{*} \mathrm{P}<0.05$, CON group versus $\mathrm{DT}$ group. $\# \mathrm{P}<0.05$, CON group versus $\mathrm{DV}$ group.

these adverse effects were recorded in our current trial and this might be attributable, in part, to a suitable dose of Dex $(0.5 \mu \mathrm{g} / \mathrm{kg})$. In addition, some rare, or less frequent, adverse side effects from the use of Dex, administered intravenously and intratracheally, might have been apparent since there were only 30 subjects in each group.

This study has some limitations which need to be considered. First, although we found that intratracheal Dex medication may have the same effect as intravenous administration in the immediate recovery period, the absorption characteristics and both pharmacokinetics and pharmacodynamics of intratracheal Dex administration still require further exploration. This was a preliminary investigation and more evidence is now required to support the wider clinical use of this method. Second, only 90 Chinese patients were enrolled in this study and a larger number of patients from multiple centres all over the world are now required to confirm the results and to record rare, or less frequent, adverse side effects from the use of Dex intratracheally. Third, the present study only tested a single dose of Dex $(0.5 \mu \mathrm{g} / \mathrm{kg})$, therefore, dose-dependent effects of intratracheal Dex on airway reflexes should be explored in the near future. Fourth, we did not collate the smoking history of our patients. The use of cigarettes should be addressed in future trials, since smoking history might also affect extubation conditions and times. Fifth, the data in our study were non-normally distributed, which was different from the retrospective results, our previous sample size calculation was not totally correct. Although the post hoc power was 0.9 and it was equal to the previous assumption, further study with larger number of patients is required to confirm the results.

\section{CONCLUSIONS}

The current study provides new insight for the perioperative use of Dex, and showed that intratracheal Dex, at a dose of $0.5 \mu \mathrm{g} / \mathrm{kg}, 30 \mathrm{~min}$ before the completion of surgery led to a smooth extubation and balanced anaesthesia recovery during gynaecological laparoscopic surgery. Thus, intratracheal Dex can be considered as an alternative method to prevent the laryngeal response emerging from general anaesthesia. 
Acknowledgements The authors acknowledge all patients for kindly participating in this study. We also thank Medjaden Bioscience for scientific editing and proofreading.

Contributors FW wrote the research protocol and oversaw the study conduct. HXZ and XYX helped conduct the study, and performed data collection. WPS and CLL performed the statistical analysis. ZPL helped write the manuscript. ZMH and CJC drafted the research protocol and wrote the manuscript.

Funding The authors have not declared a specific grant for this research from any funding agency in the public, commercial or not-for-profit sectors.

Competing interests None declared.

Patient consent Not required.

Ethics approval Institutional Review Board of the First People's Hospital of Huizhou City (approval number: 2015006).

Provenance and peer review Not commissioned; externally peer reviewed.

Data sharing statement No additional data are available.

Open Access This is an Open Access article distributed in accordance with the Creative Commons Attribution Non Commercial (CC BY-NC 4.0) license, which permits others to distribute, remix, adapt, build upon this work non-commercially, and license their derivative works on different terms, provided the original work is properly cited and the use is non-commercial. See: http://creativecommons.org/ licenses/by-nc/4.0/

(C) Article author(s) (or their employer(s) unless otherwise stated in the text of the article) 2018. All rights reserved. No commercial use is permitted unless otherwise expressly granted.

\section{REFERENCES}

1. Minogue SC, Ralph J, Lampa MJ. Laryngotracheal topicalization with lidocaine before intubation decreases the incidence of coughing on emergence from general anesthesia. Anesth Analg 2004;99:1253-7.

2. Shajar MA, Thompson JP, Hall AP, et al. Effect of a remifentanil bolus dose on the cardiovascular response to emergence from anaesthesia and tracheal extubation. Br J Anaesth 1999;83:654-6.

3. Fan $\mathrm{Q}, \mathrm{Hu} \mathrm{C}, \mathrm{Ye} \mathrm{M}$, et al. Dexmedetomidine for tracheal extubation in deeply anesthetized adult patients after otologic surgery: a comparison with remifentanil. BMC Anesthesiol 2015;15:106.

4. Bhattacharjee DP, Saha S, Paul S, et al. A comparative study of esmolol and dexmedetomidine on hemodynamic responses to carbon dioxide pneumoperitoneum during laparoscopic surgery. Anesth Essays Res 2016;10:580-4.

5. Nishino T, Hasegawa R, Ide T, et al. Hypercapnia enhances the development of coughing during continuous infusion of water into the pharynx. Am J Respir Crit Care Med 1998;157:815-21.

6. Kim H, Min KT, Lee JR, et al. Comparison of dexmedetomidine and remifentanil on airway reflex and hemodynamic changes during recovery after craniotomy. Yonsei Med J 2016;57:980-6.

7. Tanskanen PE, Kyttä JV, Randell TT, et al. Dexmedetomidine as an anaesthetic adjuvant in patients undergoing intracranial tumour surgery: a double-blind, randomized and placebo-controlled study. Br J Anaesth 2006;97:658-65.

8. Tufanogullari B, White PF, Peixoto MP, et al. Dexmedetomidine infusion during laparoscopic bariatric surgery: the effect on recovery outcome variables. Anesth Analg 2008;106:1741-8.

9. Shetty SK, Aggarwal G. Efficacy of intranasal dexmedetomidine for conscious sedation in patients undergoing surgical removal of impacted third molar: a double-blind split mouth study. J Maxillofac Oral Surg 2016;15:512-6.

10. Jee D, Park SY. Lidocaine sprayed down the endotracheal tube attenuates the airway-circulatory reflexes by local anesthesia during emergence and extubation. Anesth Analg 2003;96:293-7.

11. Solé-Lleonart C, Rouby JJ, Chastre J, et al. Intratracheal administration of antimicrobial agents in mechanically ventilated adults: an international survey on delivery practices and safety. Respir Care 2016;61:1008-14.

12. Hauber JA, Davis PJ, Bendel LP, et al. Dexmedetomidine as a rapid bolus for treatment and prophylactic prevention of emergence agitation in anesthetized children. Anesth Analg 2015;121:1308-15.
13. Steward DJ. A simplified scoring system for the post-operative recovery room. Can Anaesth Soc J 1975;22:111-3.

14. Seo H, Bang JY, Oh J, et al. Effect of Tracheal cuff shape on intracuff pressure change during robot-assisted laparoscopic surgery: the tapered-shaped cuff tube versus the cylindrical-shaped cuff tube. J Laparoendosc Adv Surg Tech A 2015;25:724-9.

15. Xu J, Zhong TD. [Comparison and superiority of streamlined liner of the pharynx airway to laryngeal mask airway or tracheal tubes for gynecological laparoscopy]. Zhonghua Yi Xue Za Zhi 2010;90:49-52.

16. Lee $\mathrm{DH}$, Park SJ. Effects of $10 \%$ lidocaine spray on arterial pressure increase due to suspension laryngoscopy and cough during extubation. Korean J Anesthesiol 2011;60:422-7.

17. Zhao G, Yin X, Li Y, et al. Continuous postoperative infusion of remifentanil inhibits the stress responses to tracheal extubation of patients under general anesthesia. J Pain Res 2017;10:933-9.

18. Greenberg MI. The use of endotracheal medication in cardiac emergencies. Resuscitation 1984;12:155-65.

19. Mussavi M, Asadollahi K, Abangah G, et al. Application of lidocaine spray for tracheal intubation in neonates - a clinical trial study. Iran $J$ Pediatr 2015;25:e245.

20. Patton JS, Fishburn CS, Weers JG. The lungs as a portal of entry for systemic drug delivery. Proc Am Thorac Soc 2004;1:338-44.

21. Kothari D, Tandon N, Singh M, et al. Attenuation of circulatory and airway responses to endotracheal extubation in craniotomies for intracerebral space occupying lesions: dexmedetomidine versus lignocaine. Anesth Essays Res 2014;8:78-82

22. Shruthi $A H$, Nethra SS, Sudheesh K, et al. Effect of dexmedetomidine on hemodynamic parameters during extubation. a prospective randomized double blind study. Middle East $J$ Anaesthesio 2016;23:457-63.

23. Bhutia MP, Rai A. Attenuation of Haemodynamic parameters in response to pneumoperitoneum during laparoscopic cholecystectomy: a randomized controlled trial comparing infusions of propofol and dexmedetomidine. J Clin Diagn Res 2017;11:UC01-UC4.

24. Chen C, Huang P, Lai L, et al. Dexmedetomidine improves gastrointestinal motility after laparoscopic resection of colorectal cancer: a randomized clinical trial. Medicine 2016;95:e4295.

25. Yang M, Zhang J, Zhang F, et al. Preoperative and intraoperative continuous use of dexmedetomidine on hyperalgesia after patients remifentanil anesthesia. Niger J Clin Pract 2017;20:244-7.

26. Yuan $Y$, Sun Z, Chen $Y$, et al. Prevention of remifentanil induced postoperative hyperalgesia by dexmedetomidine via regulating the trafficking and function of spinal NMDA receptors as well as PKC and CaMKII level in vivo and in vitro. PLoS One2017;12:e0171348.

27. Mizrak A, Erbagci I, Arici T, et al. Dexmedetomidine use during strabismus surgery in agitated children. Med Princ Pract 2011;20:427-32.

28. Lu C, Zhang LM, Zhang Y, et al. Intranasal dexmedetomidine as a sedative premedication for patients undergoing suspension laryngoscopy: a randomized double-blind study. PLoS One 2016;11:e0154192.

29. Mukherjee A, Das A, Basunia SR, et al. Emergence agitation prevention in paediatric ambulatory surgery: a comparison between intranasal dexmedetomidine and clonidine. J Res Pharm Pract 2015:4:24-30.

30. Rani P, Hemanth Kumar VR, Ravishankar M, et al. Rapid and reliable smooth extubation - Comparison of fentanyl with dexmedetomidine: a randomized, double-blind clinical trial. Anesth Essays Res 2016;10:597-601.

31. Khan ZP, Ferguson CN, Jones RM. Alpha-2 and imidazoline receptor agonists. Their pharmacology and therapeutic role. Anaesthesia 1999:54:146-65.

32. Panchgar V, Shetti AN, Sunitha HB, et al. The effectiveness of intravenous dexmedetomidine on perioperative hemodynamics, analgesic requirement, and side effects profile in patients undergoing laparoscopic surgery under general anesthesia. Anesth Essays Res 2017;11:72-7.

33. Wu X, Hang LH, Wang $\mathrm{H}$, et al. Intranasally administered adjunctive dexmedetomidine reduces perioperative anesthetic requirements in general anesthesia. Yonsei Med J 2016;57:998-1005.

34. Geng J, Qian J, Cheng H, et al. The influence of perioperative dexmedetomidine on patients undergoing cardiac surgery: a metaanalysis. PLoS One 2016;11:e0152829. 